\title{
De patito feo a cisne. Evolución para micro pymes en seguridad y salud laboral
}

\author{
From ugly duckling to swan. The way forward for \\ micro and small enterprises in occupational health \\ and safety
}

\section{Ángel Vidal}

Gerente y Fundador de Protón Electrónica SLU. España.

\author{
Correspondencia \\ Ángel Vidal \\ Pol. Ind. Aurrera, P.E. INBISA, Pab.A3, 48510 Trapagarán, Bizkaia, España \\ +34944275784 \\ ange|@protonelectronica.com
}

La salud en la empresa viene a ser un indicador, importante de la vigilancia de la Salud Pública y de sus determinantes sociales, para evitar las desigualdades en salud. El desarrollo de ésta en la empresa debe tener en cuenta el contexto socio-económico, las políticas sociales y sanitarias, las normas y valores, el estatus social del trabajador, el sistema de educación, la cohesión social, los factores biológicos y psicosociales que inciden en el sistema de atención sanitaria y la distribución a la salud y el bienestar.

Resumen

Cuando en Abril de 2011 obtuvimos nuestro primer Galardón Europeo a las Buenas Prácticas en la anterior Campaña sobre el Mantenimiento Seguro, la pregunta que más nos realizaron no fue en relación con los detalles de nuestro modelo de Coordinación Empresarial. La pregunta era: ¿Cómo una micro pyme de aquí ha conseguido ganar un premio europeo en Prevención de Riesgos? y la respuesta que dimos, un sencillo ejemplo, el nuestro, resultó ser para nuestra sorpresa nuevamente galardonado. Proponemos un modelo de Prevención de Riesgos aplicable a las pequeñas empresas, que suponen el mayor colectivo susceptible de padecer daños en la salud como consecuencia de su trabajo y que además, son las que disponen de menos recursos. Pretendemos obtener resultados excelentes con unos mínimos recursos utilizados con eficacia y eficiencia.

Palabras clave: Micro Pymes, Seguridad y Salud Laboral.

Abstract

When in April 2011 we got our first European Award for Best Practices in Campaign on Safe Maintenance, the most asked question was not about the details of our enterprises coordination model but how a micro establishment in Spain had managed to win a European award in Health and Safety. The answer that we have given is an explanation of our simple model of work. To our surprise turned out to be winning again. We propose a model of risk prevention to implement in small businesses. These enterprises are exposed to higher risks due to scarce resources. We aim to achieve excellent results despite limited financial resources.

Key words: Micro enterprises, Occupational Health and Safety. 


\section{DESCRIPCIÓN DE LA EMPRESA}

Protón Electrónica se dedica al Asesoramiento, Diseño, Comercialización, Instalación y Mantenimiento de Equipos Eléctricos y Electrónicos relacionados con la Calidad y la Eficiencia Eléctrica. Inició sus actividades en 1981 en Bilbao y en la actualidad es una micro pyme de 6 personas ubicada en Trapagarán, localidad próxima a Bilbao.

El compromiso de la Empresa con la Sostenibilidad está avalado por las certificaciones ISO9001, OHSAS18001, ISO14001 y la reciente norma de Eco Diseño ISO14006.

El liderazgo de la Dirección y la participación de nuestras Personas para promover unas condiciones de Trabajo Seguras y Saludables han sido reconocidos públicamente con dos Galardones Europeos a las Buenas Prácticas consecutivos. Concedidos por la Agencia Europea de Seguridad y Salud en el Trabajo en las Campañas 2012-2013 "Trabajando Juntos por la Prevención de Riesgos" y 2010-2011 sobre el "Mantenimiento Seguro". A los que se añaden el Premio La Fundación San Prudencio 2013 y el Premio OSALAN 2009 en Prevención de Riesgos Laborales, una Mención de Honor del Premio Nacional DIPLÓOS 2011 y una candidatura finalista al Premio Internacional ORP2011.

\section{PRL EN LA EMPRESA}

Aparentemente las necesidades en materia de PRL de la Empresa son las propias del Riesgo Eléctrico y las tareas de Mantenimiento. Sin embargo, cuando las tareas de Mantenimiento se realizan en los domicilios de los clientes, con intervenciones de corta duración y mucha disparidad de centros de trabajo, los riesgos son la suma de la propia actividad más los de todas las ubicaciones en las que se encuentran los equipos sujetos a mantenimiento.

La Dirección de la Empresa es responsable del Sistema Integrado de Gestión y cuenta con el apoyo especializado de un Servicio Ajeno de Prevención en todas las modalidades preventivas y de vigilancia de la salud.

Objetivos:

- Siniestralidad: 0 (Continuadamente vs 2012: 01 a 09 Trabajadores: 3.006).

- Absentismo < 1 (2012: 0,69 vs Sector: 6,16) poco más que alguna gripe.

- Trabajo en equipo, participación.

- Personas formadas y capacitadas.

- Buen ambiente laboral.

- Reconocimiento social.

\section{PUNTO DE PARTIDA}

Trabajadores afiliados al Régimen General y Minería del Carbón 2009. Excluidos los accidentes "in itínere" y los trabajadores "autónomos"

\begin{tabular}{lrrrr}
\hline Tamaño & Incidencia total & $\begin{array}{c}\text { Incidencia accidentes } \\
\text { leves }\end{array}$ & $\begin{array}{c}\text { Incidencia accidentes } \\
\text { graves }\end{array}$ & $\begin{array}{c}\text { Incidencia accidentes } \\
\text { mortales }\end{array}$ \\
\hline 01 a 09 trab. & $4.134,1$ & $4.079,3$ & $\mathbf{4 8 , 9}$ & $\mathbf{5 , 9}$ \\
10 a 25 trab. & $6.623,0$ & $6.554,7$ & $\mathbf{6 0 , 7}$ & $\mathbf{7 , 6}$ \\
26 a 49 trab. & $7.147,1$ & $7.085,2$ & $\mathbf{5 3 , 5}$ & $\mathbf{8 , 3}$ \\
50 a 249 trab. & $6.661,4$ & $6.618,0$ & 38,5 & 4,9 \\
250 a 499 trab. & $5.120,5$ & $5.092,3$ & 25,3 & 2,9 \\
500 a 1.000 trab. & $4.041,2$ & $4.014,3$ & 23,2 & 3,7 \\
Más de 1.000 trab. & $1.374,4$ & $1.365,8$ & 7,9 & 0,7 \\
\multicolumn{1}{c}{ Total } & $\mathbf{4 . 4 2 3 , 7}$ & $\mathbf{4 . 3 8 5 , 0}$ & $\mathbf{3 4 , 4}$ & $\mathbf{4 , 3}$ \\
\hline
\end{tabular}


Las pequeñas empresas, pymes, micro-pymes y autónomos somos "el patito feo" de la SST (Seguridad y Salud en el Trabajo). Los escasos recursos y la subcontratación de tareas con mayor riesgo originan alta siniestralidad. Casi la mitad de los trabajadores lo hacemos en empresas de menos de 50 personas.

\section{LIDERAZGO DE LA DIRECCIÓN}

En las pequeñas empresas, la propiedad suele coincidir con las funciones directivas. Además, se mantiene una relación próxima con los trabajadores a los que se conoce personalmente. No tenemos recursos para contratar a un profesional de la prevención y autorizarle a detener los trabajos delegando así la responsabilidad. No podemos decir "la dirección no me apoya" porque a nosotros la dirección siempre nos apoya. Ejemplaridad y Proporcionalidad.

Cada directivo debe encontrar los motivos que le impulsan a desear que su empresa sea un cisne en SST. Los míos son fáciles de explicar. Llevo más de 25 años trabajando con algunas de las personas de mi empresa. Hemos crecido juntos y pasado por mejores y peores momentos. Ni por un instante quisiera tener que lamentar ante sus familias y ante los demás trabajadores, una desgracia laboral que de alguna manera previsible yo hubiera podido evitar. Esa responsabilidad moral no tiene precio.

Las estadísticas europeas recientemente publicadas indican que el motivo más común es el cumplimiento de requisitos legales... evitar sanciones e incluso la cárcel. También es un motivo válido si se tiene siempre presente cuando se toman las decisiones.

\section{GESTIÓN DOCUMENTADA}

Además de ser una obligación legal, necesitas una organización documentada y las tecnologías actuales nos suministran recursos asequibles. Propongo una documentación práctica y asumida como propia, utilizada como vía de comunicación y de participación. A mí tampoco me gusta el papeleo innecesario. La redacción de una Política en la que se reconozca que la SST es una prioridad será el primer paso y realizar una encuesta para los trabajadores el siguiente.

Declararás que la SST es una de las prioridades de la dirección, que todas las personas de la empresa han sido informadas de ésta premisa, deberán colaborar en su desempeño y mejora permanente. Consecuentemente, dedicarás los recursos necesarios para que pueda ser llevada a la práctica diaria. Pon la fecha y ya tienes una Política de Seguridad y Salud en el Trabajo documentada. Puedes guiarte por las Políticas publicadas por grandes empresas o tomar como ejemplo la publicada en nuestra web, adaptándola a tu empresa y a tu manera de expresarte para hacerla tuya. Este requisito es imprescindible, puedes tomar ejemplo pero no puedes copiar sin más. La Política de SST que has redactado debe ser comunicada cómo has indicado que harías. Imprímela, fírmala y colócala en un lugar visible de la empresa.

Existe un mínimo de documentación que la normativa impone. La extensión del artículo no permite entrar en detalles, pero debes procurar que toda te resulte de utilidad y forme parte de las tareas cotidianas de la empresa. Puedes encontrar algunos de nuestros ejemplos en nuestra página www.protonelectronica.com.

\section{PARTICIPACIÓN}

Reserva una hora aproximadamente de tiempo en la que puedas reunir a los trabajadores de tu empresa. En la reunión, comunica formalmente la decisión de mejorar 
la SST, infórmales de la Política publicada y de que su colaboración es necesaria. Reparte las encuestas y solicita su cumplimentación en la propia reunión.

Pide ayuda. Algún trabajador o trabajadora que voluntariamente esté dispuesto a colaborar de un modo más intenso que el mínimo al que se supone que están obligados. Naturalmente, las horas que dedique a tareas de SST serán horas trabajadas. A falta de voluntarios, establece turnos, anuales o semestrales, empezando por los trabajadores con más antigüedad, la participación no es optativa y la SST no es una responsabilidad exclusiva de la Dirección.

Nosotros los hemos llamado "Enlaces de Prevención". La figura más conocida, el Delegado de Prevención, vinculada a la representación general de los intereses de los trabajadores, el "Delegado de Personal", precisa de más requisitos formales sin que por ello tenga necesariamente que aportar un mayor valor a la gestión de la SST. Tratamos de describir cómo hacer mucho en SST con lo mínimo. Formalizar el nombramiento en un documento y establecer los turnos, aportará credibilidad e implicación al proceso de mejora. Cada vez que sea necesario tomar una decisión relacionada con la SST reúnete con la persona voluntaria o designada y pide su opinión. Nosotros hemos necesitado hacer una ronda completa de turnos, 3 años, para tener una candidata a Delegada de Prevención que resultó elegida por unanimidad.

Revisa los resultados de las encuestas. Envía una copia al Servicio Ajeno de Prevención si lo tienes. Si tú mismo vas a asumir la gestión de la prevención por cumplir los requisitos legales vigentes, en nuestro caso sería para empresas de menos de 10 trabajadores, las encuestas serán una entrada de información complementaria para la Evaluación de Riesgos.

\section{FORMACIÓN}

La formación debe ser permanente y a todos los niveles. Con ejemplaridad, te inscribirás en un Curso para desempeñar Funciones Básicas en SST. Qué menos si vas a dirigirlas en tu empresa. Es posible que alguno de tus trabajadores ya lo tenga por exigencia de tus clientes, son cursos de entre 50 y 70 horas que habilitan para ser un "Recurso Preventivo". Si éste primer paso ya lo tienes realizado, encuentra alguna acción formativa relacionada con la actividad de tu empresa que actualice o incremente tus conocimientos. La ejemplaridad debe ser comunicada y tus trabajadores deben saber que realizas o incrementas ésta formación básica y que has tomado la decisión de mejorar la SST de tu empresa.

En línea descendente de mando o antigüedad en la empresa, todos los trabajadores deberán recibir la formación que les capacitará para el desempeño de las funciones básicas de SST y para ser "Recursos Preventivos" de sí mismos permanentemente, y de otros trabajadores cuando la situación requiera de un nombramiento formal y una dedicación de vigilancia específica. Contacta con tu Servicio Ajeno de Prevención o con las autoridades de trabajo para conocer cómo recibir la formación, el costo no será elevado y la formación es la principal materia prima de la que no podemos prescindir.

\section{RESULTADOS Y BENEFICIOS}

- Satisfacción Personal de promover y participar en una Empresa Cohesionada y Sostenible. (Motivo promotor SST personal).

- Seguridad Jurídica para los responsables. (Motivo promotor SST más votado).

- Económicamente: El que corresponde por reducción de siniestralidad y absentismo con la media del sector al que pertenezca la empresa.

- Reconocimiento social y prestigio empresarial. Dos Galardones Europeos a las Buenas Prácticas. 
Dublín, 29 de abril de 2013:

Salón de actos lleno de prensa especializada, expertos internacionales en SST, Galardonados y Menciones de Honor con sus acompañantes institucionales, Jurados, Autoridades del país anfitrión en funciones de Presidencia Europea... Se escucha:

"A escala individual, las empresas europeas no siempre reconocen las ventajas de tener unas politicas eficaces sobre salud y seguridad en el trabajo, en especial las microempresas y las pequeñas y medianas empresas", declaró Christa Sedlatschek, Directora de EU-OSHA.

"Con este premio se demuestra que incluso una empresa con sólo siete empleados puede aplicar soluciones de prevención innovadoras, como es el caso de los españoles que han conseguido uno de los galardones", añadió.

Publicado en todas las lenguas oficiales europeas. Y esos... iiéramos nosotros!!

\section{CONCLUSIONES}

La SST se puede y se debe mejorar y es responsabilidad de todos, trabajando juntos, llevar a cabo las tareas necesarias para conseguirlo. Esas tareas no solo son tangibles, también son culturales, aptitud y actitud. Yo estoy cumplimentando la parte que me toca.

Las políticas prioritariamente sancionadoras están originando miedo y elusión de responsabilidades a todos los niveles. Es necesario encontrar el medio para que los directivos de las pequeñas empresas se impliquen, participen y se sientan apoyados y tutelados. A ellos va dirigida esta propuesta, a los directivos de empresas como la nuestra, los "patitos feos" de la Prevención.

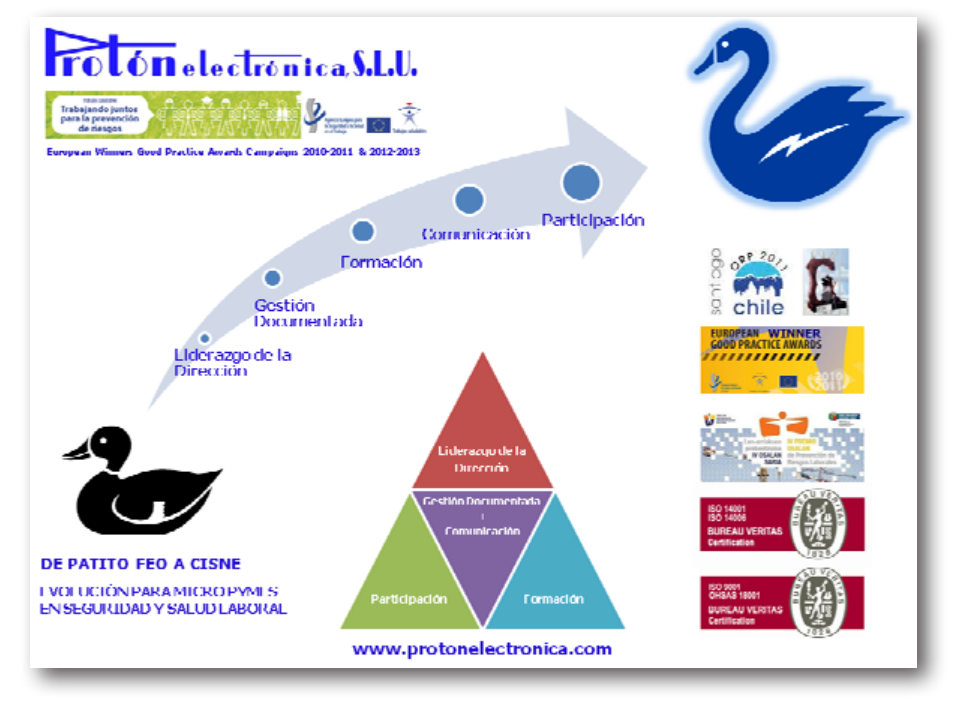

